\title{
Measurement of the direct CP violation by NA48 experiment at CERN
}

\author{
I. Mikulec ${ }^{*} \dagger$ a \\ ${ }^{a}$ CERN, CH-1211 Genève 23, Switzerland
}

The NA48 experiment at the CERN SPS accelerator aims to measure $\operatorname{Re}\left(\varepsilon^{\prime} / \varepsilon\right)$, the direct CP violation parameter in $K^{0} \rightarrow 2 \pi$ decays, to the precision of $2 \times 10^{-4}$. In the year 1999, the NA48 collaboration has published its first measurement based on the 1997 data. The presented result is a preliminary measurement from data collected in the 1998 run which contain 2.5 times more $K_{L} \rightarrow 2 \pi^{0}$ decays than the 1997 data. A combined result of $\operatorname{Re}\left(\varepsilon^{\prime} / \varepsilon\right)=(14.0 \pm 4.3) \times 10^{-4}$ has been obtained.

\section{Introduction}

Neutral kaons, $K^{0}$ and $\bar{K}^{0}$, are created in strong interactions with a defined strangeness. If quark flavour was conserved these particles would remain stable. In weak interactions however, the quark flavour is violated as characterised by the Cabbibo-Kobayashi-Maskawa (CKM) mixing matrix. In this way, through weak interactions, neutral kaons decay and the decaying states become linear combinations of strangeness eigenstates. Until 1964 it was believed that these states are $\mathrm{CP}$ eigenstates as it is required by the $\mathrm{CP}$ invariance. In 1964 Christenson, Cronin, Fitch and Turlay [1] discovered a small deviation from the CP symmetry by observing that, not only short lived kaons, but also the long lived neutral kaons decay into two pions. This proved that the decaying states do not correspond exactly to $\mathrm{CP}$ eigenstates but contain a small admixture $\varepsilon$ of the opposite CP state.

Soon after this discovery a fifth - superweak interaction was suspected to be at the origin of the CP violation [2]. The discovery of the third quark family confirmed that the violation of the CP symmetry can also be accommodated into the Standard Model through the imaginary phase in the CKM mixing matrix. Moreover, in contrary

\footnotetext{
*on behalf of the NA48 Collaboration: Cagliari, Cambridge, CERN, Dubna, Edinburgh, Ferrara, Firenze, Mainz, Orsay, Perugia, Pisa, Saclay, Siegen, Torino, Warszawa, Wien

${ }^{\dagger}$ on leave from Institut für Hochenergiephysik, Nikolsdorfergasse 18, A-1050 Wien,Austria
}

to the superweak model, Standard Model allows for two mechanisms of $\mathrm{CP}$ violation: besides the state mixing also a direct decay of the $\mathrm{CP}$ odd neutral kaon state into the $\mathrm{CP}$ even two pion state is possible. This effect, labelled direct $\mathrm{CP}$ violation, is described by the parameter $\varepsilon^{\prime}$.

First evidence of the direct $\mathrm{CP}$ violation was observed in 1993 by the NA31 experiment at CERN by measuring $\operatorname{Re}\left(\varepsilon^{\prime} / \varepsilon\right)=(23 \pm 6.5) \times 10^{-4}$ [3]. This observation was not confirmed by the experiment E731 at Fermilab which measured $\operatorname{Re}\left(\varepsilon^{\prime} / \varepsilon\right)=(7.4 \pm 5.2 \pm 2.9) \times 10^{-4}[4]$. A new generation of experiments in both laboratories was setup to clarify the situation. In 1999 both new experiments presented their results based on the first bunch of their statistics. Both confirmed the existence of the direct CP violation and definitely ruled out the superweak model. The Fermilab experiment $\mathrm{KTeV}$ measured $\operatorname{Re}\left(\varepsilon^{\prime} / \varepsilon\right)=(28.0 \pm 4.1) \times 10^{-4}[5]$ and NA48 at $\operatorname{CERN} \operatorname{Re}\left(\varepsilon^{\prime} / \varepsilon\right)=(18.5 \pm 7.3) \times 10^{-4}[6]$. The final results of these two experiments, with substantially smaller uncertainties, are expected to conclude on the size of the direct $\mathrm{CP}$ violation effect and provide a reciprocal cross-check. A third experiment, based on a very different kaon production technique - in a collider, KLOE at DA $\Phi$ NE [7], will help to accomplish this task.

The experimental determination of $\operatorname{Re}\left(\varepsilon^{\prime} / \varepsilon\right)$ is based on the fact that the two $\mathrm{CP}$ violating neu- 
tral kaon decay amplitudes into two pions

$$
\eta_{+-} \equiv \frac{A\left(K_{L} \rightarrow \pi^{+} \pi^{-}\right)}{A\left(K_{S} \rightarrow \pi^{+} \pi^{-}\right)} \simeq \varepsilon+\varepsilon^{\prime}
$$

and

$$
\eta_{00} \equiv \frac{A\left(K_{L} \rightarrow \pi^{0} \pi^{0}\right)}{A\left(K_{S} \rightarrow \pi^{0} \pi^{0}\right)} \simeq \varepsilon-2 \varepsilon^{\prime}
$$

contain different admixture of the two $\mathrm{CP}$ violating processes in charged and in neutral mode. Therefore a double ratio

$$
R=\frac{\Gamma\left(K_{L} \rightarrow \pi^{0} \pi^{0}\right)}{\Gamma\left(K_{S} \rightarrow \pi^{0} \pi^{0}\right)} / \frac{\Gamma\left(K_{L} \rightarrow \pi^{+} \pi^{-}\right)}{\Gamma\left(K_{S} \rightarrow \pi^{+} \pi^{-}\right)}
$$

is an observable sensitive to $\varepsilon^{\prime} / \varepsilon$ :

$$
\operatorname{Re}\left(\varepsilon^{\prime} / \varepsilon\right) \simeq \frac{1-R}{6}
$$

The NA48 experiment started to collect data for the $\operatorname{Re}\left(\varepsilon^{\prime} / \varepsilon\right)$ measurement in the year 1997 . This paper describes the preliminary result from the analysis of data taken in the second running period in 1998 which contains $\sim 2.5$ times more statistics than the published result based on 1997 data [6]. The improvements expected to take place in the final analysis will be pointed out.

\section{NA48 method}

The NA48 experiment was proposed in the year 1990 [8]. The aim of the experiment is to measure $\operatorname{Re}\left(\varepsilon^{\prime} / \varepsilon\right)$ to an accuracy of $2 \times 10^{-4}$ with the double ratio technique using nearly collinear simultaneous $K_{S}$ and $K_{L}$ beams.

One necessary condition to reduce the uncertainty to such a small level is to collect a sufficient amount of statistics. Around five millions of $K_{L} \rightarrow \pi^{0} \pi^{0}$, which is the statistically limiting decay mode, are needed. This requires a high intensity beam with a powerful trigger and data acquisition systems as well as a high capacity data storage and data processing farm.

Besides statistics a lot of care has to be taken to control the systematic biases. The NA48 method consists in taking the maximal benefit from the double ratio technique which allows for two fold cancellations: between the charged and the neutral mode and between the $K_{L}$ and the $K_{S}$ beams. In order to cope with time varying conditions all four decay modes are collected simultaneously. In such way beam fluxes and $K_{S}$, $K_{L}$ identification inefficiencies cancel between the charged and the neutral modes and detector, trigger and selection inefficiencies as well as losses due to accidental activity in the detector cancel between the $K_{S}$ and the $K_{L}$ beams.

In order to minimise the acceptance correction, which depends strongly on the distance of the decay vertex from the detector, all four decay modes are selected from the common decay region. Moreover, since due to the large lifetime disparity the $K_{S}$ and $K_{L}$ populate the decay volume in different ways, $K_{L}$ decays are weighted according to their decay vertex position to match the $K_{S}$ decay distribution. In this way the $K_{S}$ and the $K_{L}$ acceptances become similar and approximately cancel in the double ratio.

The only systematic effect which cannot be reduced by cancellations is the background. However, employing high resolution detectors: magnetic spectrometer for charged and liquid krypton electro-magnetic calorimeter for neutral decays, the backgrounds are suppressed to a sufficiently low level.

The technique described above, brings the double ratio of the four raw event counts very close to the final measurement. The influence of slight differences in the $K_{L}$ and the $K_{S}$ decay spectra is eliminated by averaging the double ratio in 20 bins of kaon energy. The sum of the corrections needed to account for the residual systematic biases is $<0.5 \%$.

\section{Experimental setup}

$K_{L}$ and $K_{S}$ beams are produced by the primary $450 \mathrm{GeV}$ proton beam delivered by the SPS accelerator. The beam arrives in pulses with a duration of $\sim 2.5 \mathrm{~s}$ with a period of $14.4 \mathrm{~s}$ and with an intensity of $\sim 1.4 \times 10^{12}$ protons per pulse. The neutral kaons are produced in a beryllium target of a cylindrical shape. The angle between the proton beam and the $K_{L}$ target axis is $2.4 \mathrm{mrad}$ which helps to reduce the neutron admixture in the kaon beam. Since the distance between the 


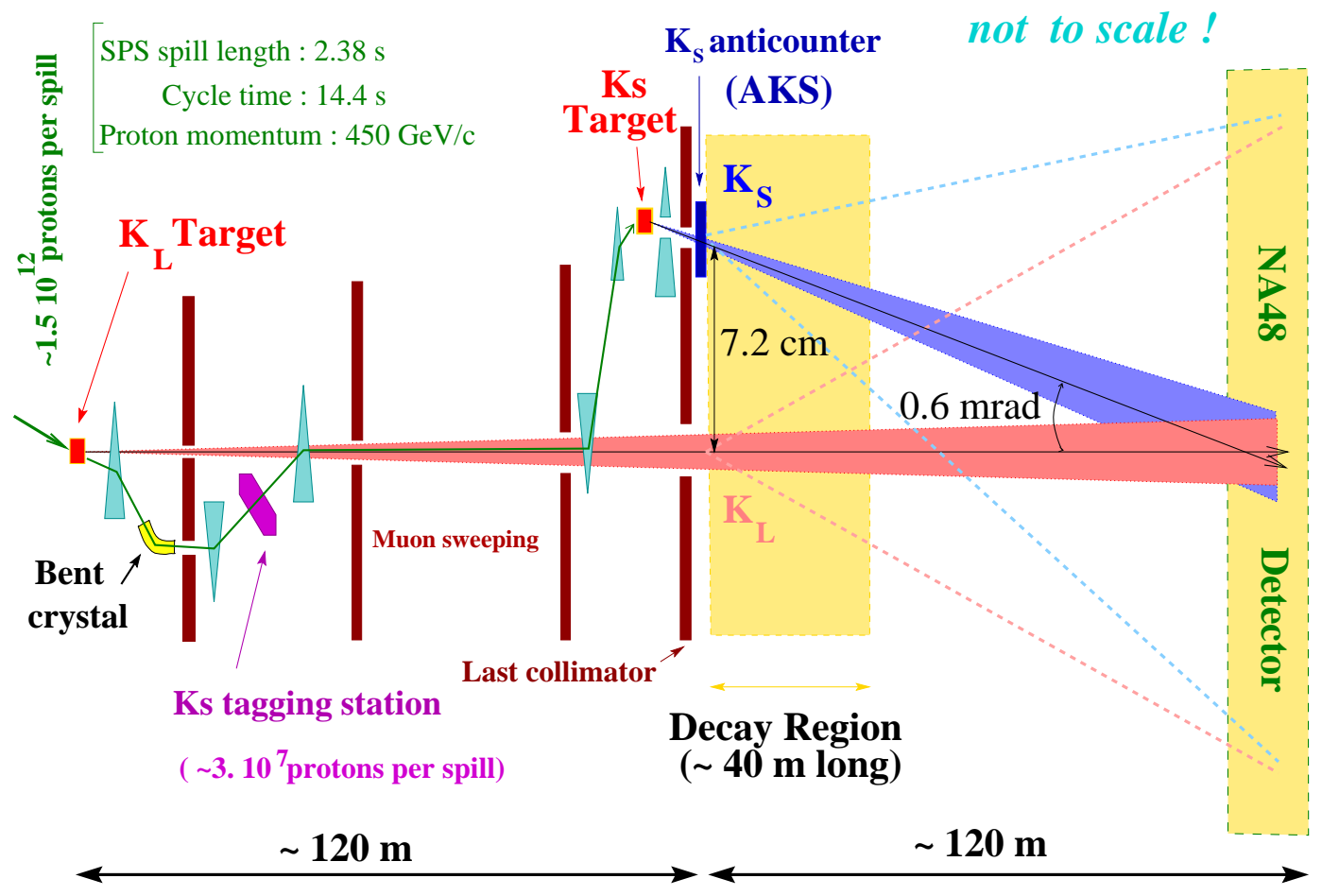

Figure 1. The layout of the NA48 experiment (side view).

$K_{L}$ target and the final collimator is $\sim 21 K_{S}$ lifetimes for average kaon energy, practically all $K_{S}$ decay before the final collimator (Fig. 1). A small effect from the interference is kept under control.

All charged particles produced at the $K_{L}$ target as well as the remaining proton beam are deflected by a sweeping magnet. A bent silicon crystal [9], deflects the proton beam back to the $K_{L}$ beam direction by channelling and at the same time attenuates the beam to $\sim 3 \times 10^{7}$ ppp which is sufficient to produce enough $K_{S} \rightarrow 2 \pi$ decays in the decay volume to match the number of CP violating $K_{L} \rightarrow 2 \pi$ decays. The $K_{S}$ target, similar to the $K_{L}$ target, is placed $7.2 \mathrm{~cm}$ above the $K_{L}$ beam $120 \mathrm{~m}$ downstream the $K_{L}$ target. The production angle in this case is 4.2 mrad, which is chosen to obtain similar between the $K_{S}$ and the $K_{L}$ decay spectra. After the $K_{S}$ target another sweeping magnet cleans the $K_{S}$ beam from charged particles. The angle between the $K_{S}$ and the $K_{L}$ beams is $0.6 \mathrm{mrad}$ and the beam axes cross at the position of the electromagnetic calorimeter.

The fact that both $K_{S}$ and $K_{L}$ beams cross each other in the detector makes it impossible to recognise the origin of $2 \pi^{0}$ decays. Therefore a tagging counter is placed in the attenuated proton beam just after the bent crystal in order to tag protons which are going to produce the $K_{S}$ beam. This is done by measuring the time of flight between the protons and the kaon decay products.

The tagging counter contains two ladders of horizontal and vertical thin scintillator plates which, seen from the beam axis, form a grid covering the whole beam profile. The plates have a varying thickness, between $200 \mu \mathrm{m}$ in the centre of the beam profile and $3000 \mu \mathrm{m}$ at the beam 
edges, to equalise the rate of the protons to $\sim 1 \mathrm{MHz}$ per counter. They are read out with the $960 \mathrm{MHz}$ sampling rate obtaining a time resolution of $\sim 200$ ps per counter and a double pulse resolution of $\sim 4$ ns. These parameters are important in order to minimise the time window for $K_{S}$ tagging and thus reduce the probability of the accidental coincidences with $K_{L}$ decay products. The good double pulse resolution is needed to avoid shifts in the proton time measurement leading to $K_{S}$ identification inefficiencies $[10,11]$.

The detector apparatus contains elements allowing to reconstruct the time of both charged and neutral decays with similar accuracy as the tagging counter. Additionally position and energy resolutions give sufficient rejection power for background and pile up events to keep the samples clean down to $0.1 \%$ level.

- The neutral decays are reconstructed based entirely on the information from the electromagnetic calorimeter. The calorimeter contains $\sim 27$ radiation lengths of liquid krypton ( $\mathrm{LKr}$ ). Its time resolution for showers above $20 \mathrm{GeV}$ is better than $300 \mathrm{ps}$ and the position resolution better than $1.3 \mathrm{~mm}$. The energy resolution is

$$
\frac{\sigma_{E}}{E}=\left[0.5 \oplus \frac{3.2}{\sqrt{E(G e V)}} \oplus \frac{10 .}{E(G e V)}\right] \%
$$

This allows a powerful rejection of the high rate background from $K_{L} \rightarrow 3 \pi^{0}$ decays.

The calorimeter has a tower structure with $\sim 13500$ cells, with a lateral size of $2 \times 2 \mathrm{~cm}^{2}$. To minimise the inhomogeneities in the response in the cases, when the photon impact is close to the anode, the angle of the ribbons with respect to the vertical plane changes at each spacer plate by $48 \mathrm{mrad}$, resulting in a "zig-zag" shape of the ribbons. In addition, the size of each cell slightly increases along the calorimeter in such way, that the axes of all cells cross in a single point at the beginning of the fiducial decay region. This makes the calorimeter projective and minimises the dependence of posi- tion and time measurements on the depth, in which the shower develops.

Due to the high rate of particles in the detector the response of the calorimeter is made fast by measuring the initial induced current, which is proportional to the deposited ionisation. This avoids a deterioration of the signal due to the pile up of signals from different particles. The dynamic range of the digitisers is increased by employing switching amplifiers which change the amplification factor depending on the pulse height [12].

- The main device for $\pi^{+} \pi^{-}$decay identification is the magnetic spectrometer. It consists of a dipole magnet and four drift chambers, two before and two after the magnet. The spatial resolution of $\sim 100 \mu \mathrm{m}$ per plane results in a momentum resolution of

$$
\frac{\sigma_{P}}{P}=[0.5 \oplus 0.009 P(G e V / c)] \% .
$$

The whole spectrometer is placed in a helium tank separated from the vacuum decay region by a thin KEVLAR window. The helium serves to equalise the pressure on the drift chamber walls from the outside, keeping the probability of particle interactions, such as multiple scattering or photon conversion, sufficiently small. The magnet has an integral field of $\int B d l=0.84 \mathrm{Tm}$, which corresponds to a transverse momentum "kick" of $265 \mathrm{MeV} / \mathrm{c}$.

Drift chambers consist of four pairs of staggered wire planes each pair having other wire orientation rotated with respect to others in steps of $45^{\circ}$ giving to the system a high redundancy. In order to assure good time measurement for charged particles a scintillator hodoscope is placed between the spectrometer and the LKr calorimeter. Its time resolution of $\sim 200 \mathrm{ps}$ for a $\pi^{+} \pi^{-}$event is important for the $K_{S}$ tagging.

The background from the semileptonic $K_{L}$ decays is rejected with the help of LKr calorimeter and muon veto counters placed 
at the downstream end of the experimental assembly.

Due to the important neutron component in the kaon beam the vacuum tank, is prolonged in a form of a thin beam pipe through the helium tank and all sub-detectors. Nevertheless, photons and electrons from three body kaon decays produce showers in the beam pipe which flood the drift chambers creating dead time in the read out electronics of $\sim 24 \%$.

The high beam intensity places strong requirements on the trigger, data acquisition and data recording systems.

- The trigger for the $\pi^{0} \pi^{0}$ decay mode represents an independent low granularity read out of the LKr calorimeter. From horizontal and vertical projections of the energy deposits in the calorimeter all necessary quantities, like total energy and decay vertex position as well as number of showers, are computed to reduce the $\sim 1 \mathrm{MHz}$ particle rate to a $\sim 2 \mathrm{kHz}$ trigger rate. Moreover all computations are done in a pipeline with constant latency of $\sim 3 \mu$ s and without any significant dead time. The inefficiency for $2 \pi^{0}$ decays is $\sim 0.1 \%$, similar for $K_{L}$ and $K_{S}$ decays and does not introduce any bias or uncertainty on the $\operatorname{Re}\left(\varepsilon^{\prime} / \varepsilon\right)$ measurement. The measurement of the efficiency is done with the help of a scintillating fibre hodoscope placed directly in the liquid krypton providing an independent shower detection device.

- The trigger for the $\pi^{+} \pi^{-}$decays consists of two levels. First level is a combination of fast signals from the hodoscope, drift chamber multiplicity and a sum of the energies from the LKr calorimeter and ironscintillator hadron calorimeter placed behind the LKr calorimeter. In the second level a processor farm computes the decay vertex position and the invariant mass from the drift chamber signals. The maximum latency of this trigger is $100 \mu \mathrm{s}$. Dead time of $<5 \%$ arises at the extraction of the data to the processor farm. The inefficiency, which was $\sim 9 \%$ during the 1997 run, was reduced, on account of several improvements, to $\sim 2.5 \%$ in the 1998 run.

The trigger decisions are collected in a trigger supervisor system which records the absolute time of the interesting event using a clock signal distributed synchronously over the entire $250 \mathrm{~m}$ length of the experiment. The trigger decision is distributed back to all read out elements in a form of a time stamp not later than $200 \mu$ s after the actual event occurrence while all data are buffered in the front-end electronics. The time stamp is transformed to an address location in the buffer. A sufficiently large time window (100 - $250 \mathrm{~ns}$ ) around this location is read out and written to the tape. The event building and data recording is performed by a scalable system of two PC-farms.

\section{Data analysis}

The strategy of the analysis follows the main principle of the NA48 method which consists in minimisation of the corrections needed to be applied to the double ratio of the raw event counts. This reduces the uncertainties following from the corrections and makes the result robust against unexpected systematic biases. The smallness of the corrections follows from the symmetry in the way the four decay samples are counted.

The key points of the analysis can be summarised as follows:

- All four modes are counted in the same kaon energy interval, $70 \mathrm{GeV}<E_{K}<170 \mathrm{GeV}$, and decay volume, $0<c \tau / c \tau_{S}<3.5$, where $c \tau$ is the proper decay time and $c \tau_{S}$ is the $K_{S}$ lifetime. The beginning of the $K_{S}$ decay volume is determined by and anti-counter placed in the $K_{S}$ beam. This reduces the sensitivity to the energy and distance scale uncertainty.

- Each occurrence of dead time in the trigger and read out is recorded in the data. This concerns $<5 \%$ dead time in the charged trigger and $\sim 24 \%$ dead time due to multiplicity overflow in the spectrometer read 
out. The recorded dead time is applied offline to the (otherwise dead timeless) neutral samples in order to equalise intensity conditions and preserve the principle of simultaneity of the event counting.

- Despite of the possibility to determine the origin of charged decays $\left(K_{S}\right.$ or $\left.K_{L}\right)$ through extrapolated vertical decay vertex position, the kaon flavour is determined by tagging in the same way it is done for neutral decays. This makes the measurement to be sensitive only to differences between neutral and charged mistagging probabilities.

- The $K_{L}$ candidates are weighted according to their proper lifetime to match the decay vertex distributions of $K_{S}$. This reduces strongly the acceptance correction (section $4.2)$.

- Samples are counted and corrections on backgrounds, $K_{S} / K_{L}$ trigger efficiency difference, $\pi^{+} \pi^{-} / \pi^{0} \pi^{0}$ mistagging difference and $K_{S} / K_{L}$ acceptance difference are applied in bins of kaon energy in order to eliminate the effect of $K_{S} / K_{L}$ spectra difference.

- Data selection and evaluation of corrections is always cross-checked by an independent analysis to avoid bugs and misinterpretation of the analysis methods.

\section{1. $K_{S}$ tagging}

In order to associate each $2 \pi$ candidate with the one of the two beams, protons destined for the $K_{S}$ target are registered in the tagging counter. In case of a time coincidence between the detector signals from decay products and one of the tagged protons, taking into account the appropriate time offsets, the decay is attributed to the $K_{S}$ beam. Two ways of mistagging must be considered (Fig. 2):

- tagging inefficiency $\left(\alpha_{S L}\right)$ where at least one of the two times was mismeasured,

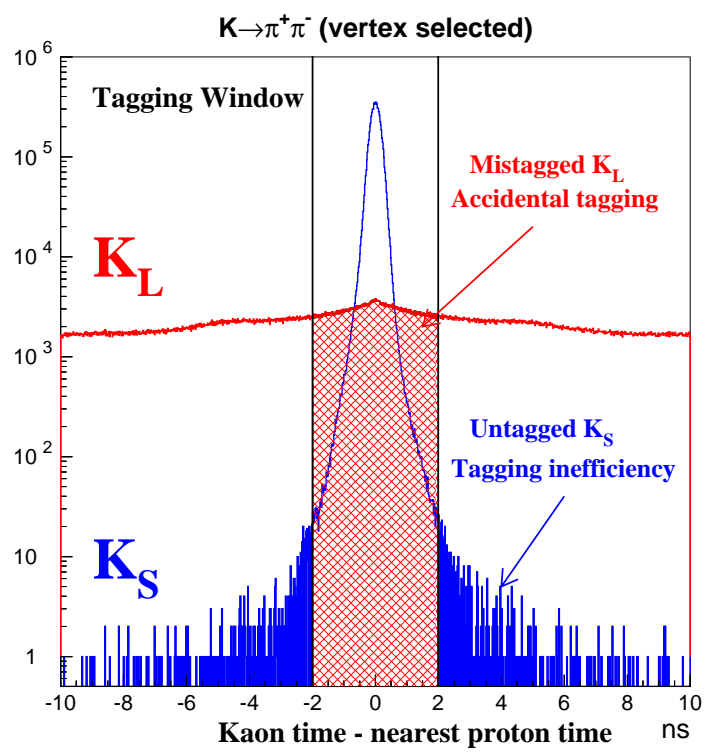

Figure 2. The distribution of the difference between the time of a $K \rightarrow \pi^{+} \pi^{-}$candidate and the nearest proton time detected by the tagging counter. The $K_{L}$ and $K_{S}$ contributions are separated by track extrapolation.

- accidental tagging $\left(\alpha_{L S}\right)$ due to accidental coincidences between protons in the tagging counter and $K_{L}$ decays.

In the charged mode, these two probabilities can be measured directly by identifying the beam from the extrapolated transversal vertex position. The extrapolation resolution and beam separation are sufficient to determine the kaon flavour with negligible uncertainty. However, in the $\operatorname{Re}\left(\varepsilon^{\prime} / \varepsilon\right)$ measurement both, charged and neutral, modes are treated symmetrically. Therefore the $\operatorname{Re}\left(\varepsilon^{\prime} / \varepsilon\right)$ measurement is sensitive only to differences between the charged and neutral mistagging probabilities:

$$
\begin{aligned}
& \Delta R_{S L} \simeq 6\left(\alpha_{S L}^{00}-\alpha_{S L}^{+-}\right) \\
& \Delta R_{L S} \simeq 2\left(\alpha_{L S}^{00}-\alpha_{L S}^{+-}\right)
\end{aligned}
$$


A coincidence window of \pm 2 ns was chosen in order to minimise the total systematic uncertainty from both ways of mistagging.

The tagging inefficiency measured in the charged mode is $\alpha_{S L}^{+-}=(1.97 \pm 0.05) \times 10^{-4}$. The dominant source of the inefficiency is the tagging counter itself which means that the major part of the inefficiency is symmetric between the charged and the neutral mode. In order to evaluate the differential effect the time measurements of scintillator hodoscope and LKr calorimeter were compared directly in $3 \pi^{0}$ decays with one photon conversion: $\left|\alpha_{S L}^{+-}-\alpha_{S L}^{00}\right|<0.5 \times 10^{-4}$, which translates to uncertainty on the double ratio of $\Delta R<3 \times 10^{-4}$. This measurement was cross-checked by direct measurement of $\alpha_{S L}^{00}$ in special runs where the $K_{L}$ beam was stopped as well as with $K_{S} \rightarrow \pi^{0} e^{+} e^{-} \gamma$ decays which, however, give a statistically less significant result.

The value of accidental tagging probability measured with charged decays is $\alpha_{L S}^{+-}=(11.05 \pm$ $0.01) \%$. The measurement of $\alpha_{\mathrm{LS}}^{00}-\alpha_{\mathrm{LS}}^{+-}$, which is the quantity the double ratio has to be corrected for (Eq. 5), is measured in two steps:

$$
\alpha_{\mathrm{LS}}^{00}-\alpha_{\mathrm{LS}}^{+-}=\left(\beta_{\mathrm{tag}}^{00}-\beta_{\mathrm{tag}}^{+-}\right)+\left(\mathrm{W}^{00}-\mathrm{W}^{+-}\right)
$$

where $\beta_{\mathrm{tag}}$ is the probability to detect a proton in a coincidence window intentionally shifted with respect to the true coincidence window. The index "tag" means that it is measured for events already identified as $K_{L}$ by the tagging counter, that is, there is no proton in the true coincidence window by definition. In order to increase the statistical significance four windows with different time offsets were used. This is possible because all protons hitting the tagging counter in $\sim 100 \mathrm{~ns}$ around the trigger are recorded in the data. The quantity $\mathrm{W}$ compensates for the difference between the shifted and the true coincidence windows as well as for the difference between the rates seen by tagged and true $K_{L}$ decays. The parameter $\beta_{\mathrm{tag}}^{00}$ is the only one which can be determined directly from $K_{L} \rightarrow \pi^{0} \pi^{0}$ decays. The quantity $\mathrm{W}^{00}$ is measured using $K_{L} \rightarrow \pi^{0} \pi^{0} \pi^{0}$ decays assuming $\mathrm{W}^{00}=\mathrm{W}^{000}$. Measurements $\beta_{\mathrm{tag}}^{00}-\beta_{\mathrm{tag}}^{+-}=(5.3 \pm 1.6) \times 10^{-4}$ and $\mathrm{W}^{00}-\mathrm{W}^{+-}=(-5.0 \pm 3.9) \times 10^{-4}$ result in the correction on double ratio of $\Delta \mathrm{R}=(1 \pm 8) \times 10^{-4}$. The uncertainty on this correction is dominated by the statistical error on $\mathrm{W}^{000}$ which reflects the small amount reconstructed $K_{L} \rightarrow \pi^{0} \pi^{0} \pi^{0}$ decays available for the preliminary result. For the final result an enhanced $3 \pi^{0}$ sample, re-filtered from the raw data, will allow to reduce the error on this correction approximately by a factor of two.

\subsection{Acceptance and proper time weight- ing}

Due to the large differences between the two neutral kaon lifetimes and between the distances of the two targets from the decay volume the proportion of $K_{S}$ and $K_{L}$ decays varies strongly within the common decay region. Since the geometrical acceptance is a function of the distance between the decay vertex and the detector this results in a large acceptance correction on the double ratio of up to $10 \%$ depending on the kaon energy. In order to avoid such a large correction, which would require a strong confidence in the precision of the detector description in the acceptance Monte Carlo, the $K_{L}$ candidates are weighted with a factor



where $\tau$ is the proper life time measured either from the $K_{S}$ or from the $K_{L}$ target and

$$
\begin{aligned}
& I_{2 \pi}(\tau)=e^{-\frac{\tau}{\tau_{S}}}+|\eta|^{2} e^{-\frac{\tau}{\tau_{L}}}+ \\
& 2|\eta| D_{P} e^{-\frac{\tau}{2}\left(\frac{1}{\tau_{L}}+\frac{1}{\tau_{S}}\right)} \cos (\Delta M \tau-\phi)
\end{aligned}
$$

is the expected $2 \pi$ decay rate. The factor $D_{P}$ is the $K^{0}-\overline{K^{0}}$ production asymmetry at the target, and $\tau_{L}, \tau_{S}, \Delta M, \phi$ are well known parameters of the neutral kaon system. Due to such weighting the ratio of the effective event counts between $K_{S}$ and $K_{L}$ becomes independent on the decay position and the acceptance correction reduces to a value of $\Delta \mathrm{R}=\left(+31 \pm 6_{\text {stat } M C} \pm 6_{\text {syst }}\right) \times 10^{-4}$ (Fig. 3). The remaining correction reflects mainly the fact that the axes of both beams are not perfectly identical. The advantage of small acceptance correction is, however, payed by a reduction of the statistical significance of the $K_{L}$ samples. 




Figure 3. The acceptance correction in bins of kaon energy before and after proper time weighting.

The increase of the statistical uncertainty in the result is $\sim 35 \%$. The systematic uncertainty on the acceptance correction is conservative and, for the final result, is likely to be improved along with increase of the Monte Carlo statistics.

\subsection{Backgrounds}

The dominant source of background in the $\pi^{0} \pi^{0}$ mode are $K_{L} \rightarrow \pi^{0} \pi^{0} \pi^{0}$ decays. This background is suppressed mainly by demanding no additional shower of more than $1.5 \mathrm{GeV}$ within $3 \mathrm{~ns}$ around the $\pi^{0} \pi^{0}$ candidate. The remaining background with two photons lost or overlapping is suppressed by an invariant mass cut applied in the space of two $\pi^{0}$ invariant masses, $m_{1}$ and $m_{2}$, using a $\chi^{2}$-like variable

$$
R_{\text {ellipse }}=\left[\frac{\frac{\left(m_{1}+m_{2}\right)}{2}-m_{\pi^{0}}}{\sigma_{+}}\right]^{2}+\left[\frac{\frac{\left(m_{1}-m_{2}\right)}{2}}{\sigma_{-}}\right]^{2}
$$

with $\sigma_{+}$and $\sigma_{-}$being the corresponding resolutions parametrised as a function of minimum photon energy in the event which is the determining parameter. The invariant mass is measured from the energies $\left(E_{i}\right)$ and positions $\left(x_{i}, y_{i}\right)$ of the showers

$$
m_{\gamma \gamma}=\frac{\sqrt{E_{1} E_{2}\left[\left(x_{1}-x_{2}\right)^{2}+\left(y_{1}-y_{2}\right)^{2}\right]}}{d_{\text {vertex }}},
$$

where the distance of the vertex $d_{\text {vertex }}$ from the detector is computed using the kaon mass $\left(m_{K}\right)$ constraint

$$
\begin{aligned}
& d_{\text {vertex }}= \\
& \frac{\sqrt{\sum_{i, j, i>j}^{4} E_{i} E_{j}\left[\left(x_{i}-x_{j}\right)^{2}+\left(y_{i}-y_{j}\right)^{2}\right]}}{m_{K}} .
\end{aligned}
$$

The invariant mass cut is applied at $R_{\text {ellipse }}<13.5$ which corresponds to 3.7 standard deviations.

The remaining background is estimated from the control region of the $R_{\text {ellipse }}$ distribution (Fig. 4) after subtracting the tail common to both $K_{S}$ and $K_{L}$ decays. The extrapolation factor was evaluated by Monte Carlo simulation. The remaining background to signal ratio in the $K_{L} \rightarrow \pi^{0} \pi^{0}$ sample amounts to $(6.6 \pm 2.0) \times 10^{-4}$. The error is dominated by the statistical uncertainty of the Monte Carlo extrapolation factor. The size of this background would be around four times larger without proper life time weighting which is due to accumulation of the background events at the downstream end of the decay region.

In the $\pi^{+} \pi^{-}$mode the two semileptonic 3body $K_{L}$ decay channels, $K_{L} \rightarrow \pi e \nu\left(K_{e 3}\right)$ and $K_{L} \rightarrow \pi \mu \nu\left(K_{\mu 3}\right)$, dominate the background. The $K_{e 3}$ channel is suppressed by the electron identification comparing the energy in the LKr calorimeter (E) with momentum from the spectrometer $(\mathrm{p})$ and requiring $\mathrm{E} / \mathrm{p}<0.8$. In order to symmetrise losses of $\pi^{+} \pi^{-}$candidates this cut is applied to $K_{S}$ sample as well. In case of $K_{\mu 3}$ no associated hit in muon veto counters closer than 4 ns is required. The remaining background is subtracted by studying the distributions of well identified $K_{e 3}$ and $K_{\mu 3}$ samples in the 2-dimensional space of invariant mass and missing transverse momentum. Two control regions are chosen, one 


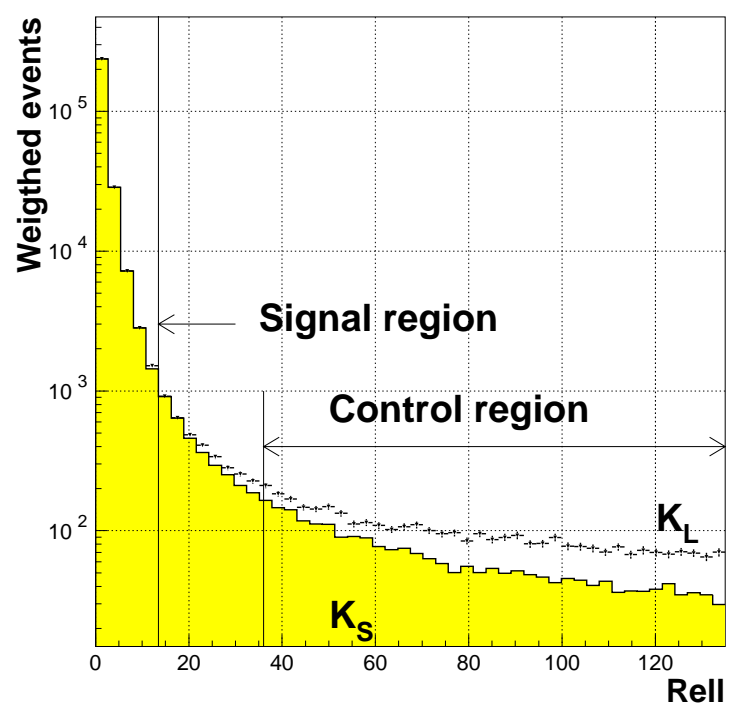

Figure 4. Distribution of the $R_{\text {ellipse }}$ variable for both $K_{S} \rightarrow \pi^{0} \pi^{0}$ and $K_{L} \rightarrow \pi^{0} \pi^{0}$ candidates. The excess in the $K_{L}$ tail is due to $K_{L} \rightarrow \pi^{0} \pi^{0} \pi^{0}$ background.

dominated by $K_{e 3}$ and one by $K_{\mu 3}$ decays. Again, the tail common to both $K_{S}$ and $K_{L} \rightarrow \pi^{+} \pi^{-}$ is subtracted. In order to symmetrise the transverse momentum resolution between the $K_{S}$ and $K_{L}$ samples, which differ due to different target positions, a rescaled variable $p_{T}^{\prime}$ is used. Finally the relative weight of $K_{e 3}$ and $K_{\mu 3}$ backgrounds in the residual $K_{L}$ tail is found and used to extrapolate to the signal region resulting in a background to signal ratio of $(19 \pm 3) \times 10^{-4}$ (Fig. 5).

In Fig. 5 at $p_{T}^{\prime 2}>0.0025(\mathrm{GeV} / \mathrm{c})^{2}$ one can see that the $K_{L}$ tail is dominated by events from re-scattered of beam particles at the collimators. These events, being $K_{S}$ decays rejected in the charged mode due to missing transverse momentum cut, remain in the neutral sample. Their amount was evaluated by reconstructing the invariant mass of $\pi^{+} \pi^{-}$candidates at high $p_{T}^{\prime}$ and subtracting the underlying background. Taking into account the difference in the charged and

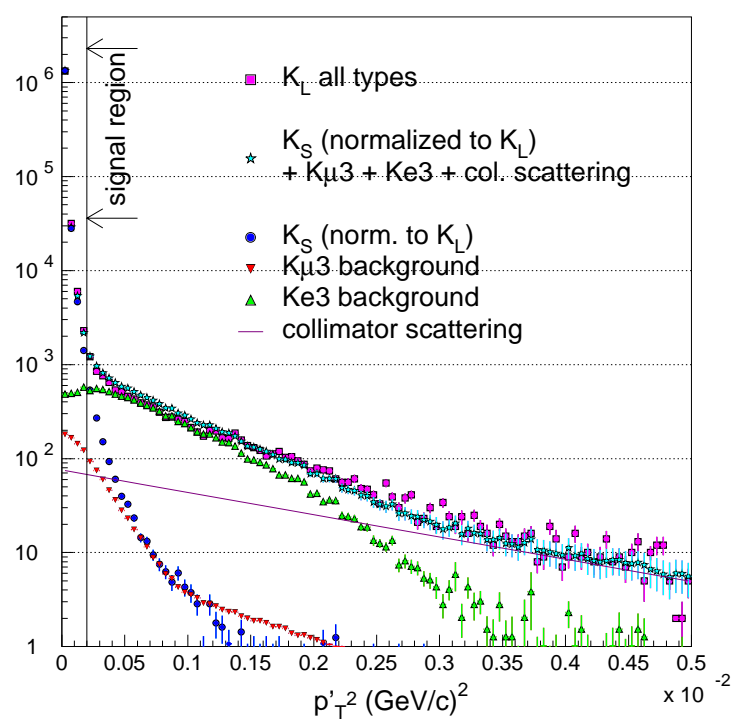

Figure 5. The distribution of missing transverse momentum ${p_{T}^{\prime}}^{2}$ in charged samples with the background extrapolation.

neutral selection a correction on double ratio of $(-10 \pm 3) \times 10^{-4}$ has been applied. This result was cross-checked with the $\pi^{0} \pi^{0}$ sample at large centre of gravity.

\subsection{Other systematics}

One of the basic systematic uncertainties on the measurement of $\operatorname{Re}\left(\varepsilon^{\prime} / \varepsilon\right)$ is the uncertainty on the distance scale. It enters the measurement through the definition of the decay region. In the case of $K_{L}$ decays no bias is introduced because the distribution of these events as a function of decay vertex position is approximately flat and gains on one side of the decay region are compensated by losses on the other side. This is not the case for $K_{S}$ decays with the characteristic exponential distribution. In order to avoid strong sensitivity to the distance scale uncertainty the beginning of the decay region is defined by an anticounter, placed in the $K_{S}$ beam, instead of using the reconstructed vertex position. The only re- 
maining place the distance scale uncertainty matters is the downstream end of the $K_{S}$ decay region. This is chosen far enough ( $3.5 K_{S}$ lifetimes) to reduce the sensitivity of double ratio to $60 \%$ of the relative uncertainty on the distance scale.

In charged decays the distance scale is defined by the knowledge of the geometry of the first two drift chambers which are used to extrapolate tracks to the vertex. Fitting the upstream edge of the $K_{S} \rightarrow \pi^{+} \pi^{-}$distribution after rejecting all events decaying before the $K_{S}$ anti-counter a correction on double ratio of $(2 \pm 2) \times 10^{-4}$ was determined.

In the neutral decay mode the distance scale is directly related to the energy scale. This is calibrated again by fitting the upstream edge of the $K_{S} \rightarrow \pi^{0} \pi^{0}$ vertex distribution. The accuracy of this calibration is $5 \times 10^{-4}$ which translates to an uncertainty of $3 \times 10^{-4}$ on the double ratio.

Apart from the energy scale, a range of other systematic biases connected to the readout of the electro-magnetic calorimeter and the neutral reconstruction, influence the result. They are summarised in the Tab. 1. These results are based on studies of the $\pi^{0} \pi^{0}$ sample and on comparing the response of LKr calorimeter and of the spectrometer for electrons from $K_{e 3}$ decays $(\mathrm{E} / \mathrm{p})$. Additionally special runs were taken regularly using $\pi^{-}$beam with two polyethylene targets producing high statistics of $\pi^{0}, \eta \rightarrow \gamma \gamma$ decays which can be used to probe the energy scale, linearity and homogeneity of the LKr calorimeter. The combined uncertainty of the neutral reconstruction systematics on the double ratio is $<10 \times 10^{-4}$. An effect of a small discrepancy between the reconstructed $\pi^{0}$ and $\eta$ masses resulting from the $\pi^{-}$runs mentioned above is added to this estimate. Among the uncertainties from the Tab. 1 the uncertainty from the non-linearity $(0.2 \%)$ in the energy measurement and the effect of nonGaussian tails play the most important role. In the final result some improvements are expected from better alignment of the drift chambers and the LKr calorimeter (E/p systematics) and from introduction of parametrised non-Gaussian tails into acceptance Monte Carlo.

A special care has to be given to losses and gains in the event counts due to accidental activ-
Table 1

Summary of uncertainties on the double ratio from the neutral reconstruction systematics.

\begin{tabular}{|l|r|}
\hline Source & $\Delta \mathrm{R}\left(\right.$ Units $\left.=10^{-4}\right)$ \\
\hline Non linearity & \pm 5 \\
Non Gaussian tails in $\sigma_{E}$ & \pm 5 \\
Transverse scale & \pm 3 \\
Inter-calibration & \pm 3 \\
Distance/Energy scale & \pm 3 \\
Other sources & \pm 4 \\
\hline
\end{tabular}

ity in the detector which comes mainly from the $K_{L}$ beam and from the detector noise. Two different effects have to be evaluated. One, due to difference in the illumination of the detector, caused by the difference in the $K_{S}$ and $K_{L}$ beam geometry and second, due to variation of the $K_{S} / K_{L}$ beam intensity ratio with time (r.m.s. of $10 \%$ ) which, along with time variations of e.g. noise or asymmetric losses between the charged and the neutral mode, can cause a bias on the double ratio.

The illumination effect was evaluated by overlaying the data samples by random events, triggered proportionally to the intensity, and results in a correction on the double ratio of $(2 \pm 6) \times 10^{-4}$. The rest of the effects is absorbed in a limit of $<10 \times 10^{-4}$ estimated based on the knowledge about the size of the dominant contributing effects. Using overlay technique with the Monte Carlo sample to avoid the doubling of the noise and more precise studies of the two beam correlation and time instabilities in the detector a significant improvement of these uncertainties is expected.

\section{Result}

The statistics collected in 1998 run is shown and compared with the 1997 statistics in the Tab. 2. The list of all corrections applied to the raw double ratio and of all systematic uncertainties is given in the Tab. 3. The size of the systematic uncertainty on the charged trigger efficiency is given by the statistics of the control sample used to measure the efficiency. Since it scales with 
Table 2

Comparison of the statistics accumulated in the 1997 and 1998 runs. The numbers are obtained after taking into account the accidental tagging.

\begin{tabular}{|c|c|c|}
\hline & \multicolumn{2}{|c|}{ Statistics in millions } \\
\hline & 1997 & 1998 \\
\hline$K_{S} \rightarrow \pi^{+} \pi^{-}$ & 2.09 & 7.46 \\
$K_{L} \rightarrow \pi^{+} \pi^{-}$ & 1.07 & 4.87 \\
$K_{S} \rightarrow \pi^{0} \pi^{0}$ & 0.98 & 1.80 \\
$K_{L} \rightarrow \pi^{0} \pi^{0}$ & 0.49 & 1.14 \\
\hline Stat. error on R & $27.0 \times 10^{-4}$ & $17.3 \times 10^{-4}$ \\
\hline
\end{tabular}

the accumulated statistics it will improve in the result from 1999 data. Moreover, due to an improvement in the efficiency and due to a change of the down-scaling of the control trigger, additional reduction of the uncertainty is expected. Other four dominant systematic uncertainties, on neutral reconstruction, on acceptance and on accidental tagging, are likely to be improved as explained in the previous section.

Table 3

Corrections and systematic uncertainties on the double ratio in the 1997 and the preliminary 1998 results. In order to obtain the effect on $\operatorname{Re}\left(\varepsilon^{\prime} / \varepsilon\right)$ the numbers must be scaled down by factor 6 (Eq. 4).

\begin{tabular}{|c|rr|rr|}
\hline & \multicolumn{4}{|c|}{$\Delta \mathrm{R}$ (Units $=10^{-4}$ ) } \\
\hline Source & \multicolumn{2}{|c|}{1997} & \multicolumn{2}{|c|}{1998 (prel.) } \\
\hline Charged trigger & +9 & \pm 23 & -1 & \pm 11 \\
Accidental tagging & +18 & \pm 9 & +1 & \pm 8 \\
Tagging efficiency & - & \pm 6 & - & \pm 3 \\
Neutral rec. syst. & - & \pm 12 & - & \pm 10 \\
Charged vertex & - & \pm 5 & +2 & \pm 2 \\
Acceptance & +29 & \pm 12 & +31 & \pm 9 \\
Neutral BKG & -8 & \pm 2 & -7 & \pm 2 \\
Charged BKG & +23 & \pm 4 & +19 & \pm 3 \\
Beam scattering & -12 & \pm 3 & -10 & \pm 3 \\
Accid. activity & -2 & \pm 14 & +2 & \pm 12 \\
\hline & & & & \\
Total & +57 & \pm 35 & +37 & \pm 24 \\
& & & & \\
\hline
\end{tabular}

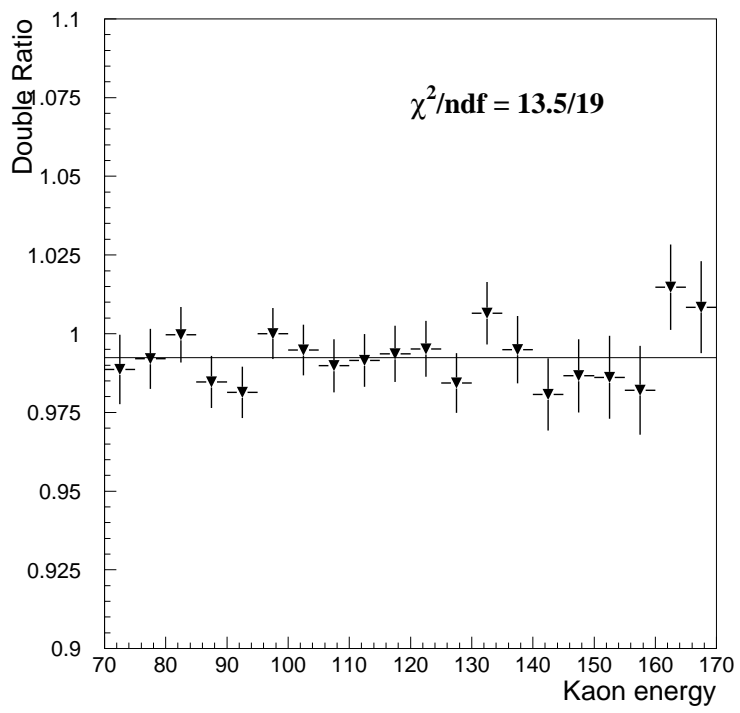

Figure 6 . The stability of the corrected double ratio with kaon energy.

The stability of the corrected double ratio with changes of the beam and detector conditions, cut variables and kaon energy (Fig. 6) was checked extensively. The preliminary result obtained from the data collected in the year 1998 is

$$
\operatorname{Re}\left(\varepsilon^{\prime} / \varepsilon\right)=\left(12.2 \pm 2.9_{\text {stat }} \pm 4.0_{\text {syst }}\right) \times 10^{-4} .
$$

The combined 1997 [6] and 1998 result is

$$
\operatorname{Re}\left(\varepsilon^{\prime} / \varepsilon\right)=(14.0 \pm 4.3) \times 10^{-4} .
$$

Small correlation in the systematic errors is taken into account.

\section{Conclusions and outlook}

The result presented in this paper confirms the existence of the direct $\mathrm{CP}$ violation with a positive value of the $\varepsilon^{\prime}$ parameter. The uncertainty on this preliminary result is expected to improve after investing more work in data checks and Monte 
Carlo studies and exploiting better the available control samples. In 1999 another $\sim 2$ million of $K_{L} \rightarrow \pi^{0} \pi^{0}$ and adequate amount of other three decay modes were accumulated. This promises to achieve a total uncertainty on the $\operatorname{Re}\left(\varepsilon^{\prime} / \varepsilon\right)$ measurement of $\lesssim 3 \times 10^{-4}$.

Due to an the implosion of the beam pipe in the spectrometer damaging all four drift chambers, the final run planned for the year 2000 had to be postponed to the year 2001. A part of the 2000 data taking period was, nevertheless, devoted to checks on accidental tagging and neutral reconstruction systematics which could be done without the magnetic spectrometer.

Taking into account the three most precise results published up to now [3-5] and the combined NA48 result the world average is

$$
\operatorname{Re}\left(\varepsilon^{\prime} / \varepsilon\right)=(19.3 \pm 2.4) \times 10^{-4} .
$$

However the $\chi^{2}=10.5 / 3$ indicates a very weak consistency among the published results. New results with better accuracy from both $\mathrm{KTeV}$ and NA48 as well as first results from KLOE should help to clarify this situation.

Unfortunately, the uncertainties of present theoretical predictions do not allow yet to draw conclusions about the compatibility of present experimental results with the Standard Model. Some new theoretical approaches were triggered by the relatively large value of the experimental world average with respect to most of the theoretical calculations of the last decade $[15,16]$. Moreover, the experimental results on $\operatorname{Re}\left(\varepsilon^{\prime} / \varepsilon\right)$ provide constraints for a variety of supersymmetric models [17].

\section{REFERENCES}

1. J.H. Christenson, J.W. Cronin, V.L. Fitch and R. Turlay, Phys. Rev. Lett. 13, (1964) 138.

2. L. Wolfenstein, Phys. Rev. Lett. 13, (1964) 562.

3. G.D. Barr et al., Phys. Lett. B 317, (1993) 233.

4. L.K. Gibbons et al., Phys. Rev. Lett. 70, (1993) 1203.
5. A. Alavi-Harati et al., Phys. Rev. Lett. 83, (1999) 22.

6. V. Fanti et al., Phys. Lett. B 465, (1999) 335.

7. F. Bossi, these proceedings.

8. G.D. Barr et al., CERN/SPSC/90-22/P253, (1990).

9. N. Doble et al., Nucl. Instrum. Methods B 119, (1996) 181.

10. P. Grafström et al., Nucl. Instrum. Methods A 344, (1994) 487.

11. H. Bergauer et al., Nucl. Instrum. Methods A 419, (1998) 623.

12. B. Hallgren et al., Nucl. Instrum. Methods A 414, (1998) 680.

13. S. Anvar et al., Nucl. Instrum. Methods A 419, (1998) 686.

14. B. Gorini et al., IEEE Trans. Nuc. Sci. 45, (1998) 1771.

15. M. Ciuchini, these proceedings.

16. M. Fabrichesi, these proceedings.

17. A. Masiero, these proceedings. 